\title{
Stacked planar optics: an application of the planar microlens
}

\author{
Kenichi Iga, Masahiro Oikawa, S. Misawa, Junichi Banno, and Y. Kokubun
}

\begin{abstract}
In this paper we present a new development of the distributed-index planar microlens of $2.5-3-\mathrm{mm}$ focal length and 0.15 N.A. and also show that it is usable with optical components in fiber communications. We then propose a concept of stacked planar optics consisting of a stack of arrayed planar microlenses and other optical components. From this configuration a 2-D array of optical devices is monolithically available, and many discrete components can be separated from the array. An optical tap array has been fabricated as an example of stacked planar optics.
\end{abstract}

\section{Introduction}

Great progress in optical fiber communications has been made and many working systems have been installed. As for the optical components used in optical fiber communication systems, three types have been considered: (1) microoptics ${ }^{1-3}$ which consists of microlenses such as gradient-index lenses or tiny spherical lenses, (2) optical fiber circuits ${ }^{4}$ which are made from manufactured fibers, and (3) integrated optics. ${ }^{5}$ There have been a lot of problems such as optical alignment and fabrication process in the first two schemes, and the integrated optics devices are still far from the usable level.

We have proposed a new stacked planar optics concept to overcome these problems. ${ }^{6}$ The stacked planar optics we detail here consists of planar and 2-D arrayed optical components such as microlenses, filters, mirrors, and we stack them in tandem to achieve functional optical components, for example, optical taps, branches, directional couplers, wavelength multiplexers/demultiplexers, and other possible components including active devices.

One of the key components in stacked planar optics is believed to be a 2-D microlens array, but no such array has been formed on a planar substrate. We have reported the fabrication of planar microlenses.7-9 A similar component is the plasma-deposited microlens reported by Khoe et al. ${ }^{10}$ The merit of the plasmadeposited lens is to have a large numerical aperture.

The authors are with Tokyo Institute of Technology, 4259 Nagatsuta, Midori-ku, Yokohama 227, Japan.

Received 2 March 1982.

0003-6935/82/193456-05\$01.00/0.

(C) 1982 Optical Society of America.
Recently we succeeded in making a microlens array that might be used in accepting light from optical fibers, ${ }^{6}$ i.e., the focal length of each individual $1.0-\mathrm{mm}$ diam lens is $2.5-3 \mathrm{~mm}$ and the maximum numerical aperture is nearly 0.17 . Since the associated acceptance angle is $20^{\circ}$, we believe this claim is reasonable. Moreover, on the basis of further work we can say that the realizable N.A. of a single lens is 0.24 and it could be made as high as 0.35 by stacking two lenses.

\section{Planar Microlens}

To realize the stacked planar optics, all optical devices must have a planar structure. The array of microlenses on a planar substrate is required to focus and collimate the light in optical circuits. We developed a planar microlens ${ }^{7-9}$ fabricated by the selective diffusion of a dopant into the planar substrate through a mask as shown in Fig. 1(a). Recently we successfully made an array of planar microlenses with 2.5-3-mm focal length (in air), and a minimum numerical aperture of 0.15 with its maximum of 0.17 , obtained experimentally from the acceptance angle. ${ }^{6}$ This planar microlens was fabricated by using an electromigration technique. ${ }^{9}$ The substrate is a $40-\times 40-\times 3-\mathrm{mm}^{3}$ planar glass where planar microlenses were formed as an $11 \times 17$ matrix with 2 -mm pitch. The radius of the mask is $40 \mu \mathrm{m}$ and the diameter of the resultant lens was $0.9 \mathrm{~mm}$. The depth of the diffused region is $\sim 0.45 \mathrm{~mm}$ which is nearly equal to the radius of the resulting lens. The improvement of focal length and N.A. is due to the better choice of mask radius $\left(r_{m}=40 \mu \mathrm{m}\right)$ and migration time $\left(T_{e}=6 \mathrm{~h}\right)$ and the reduction of leak current through the edge of the substrate.

In Fig. 1(b) an interference pattern which shows the index profile of the planar microlens is presented. The interference pattern was obtained from a $100-\mu \mathrm{m}$ thick sliced plate, including a center axis at the meridional plane $M$ in Fig. 1(a). The pattern was observed by an 

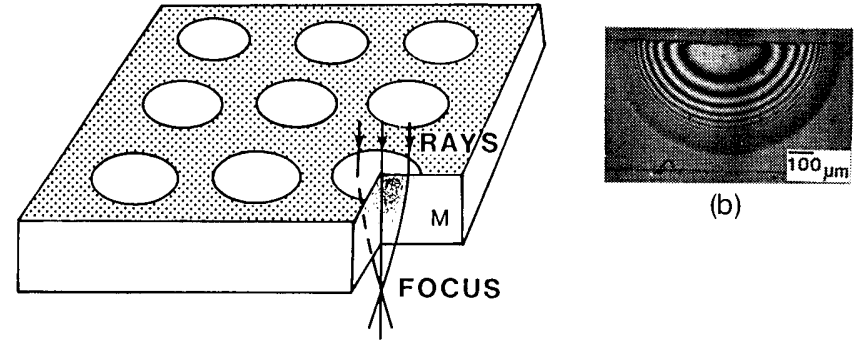

(b)

(a)

Fig. 1. Planar microlens: (a) configuration; (b) interference pattern which shows the index profile in plane $M$.

interference microscope ${ }^{11}$ with $0.65-\mu \mathrm{m}$ wavelength light source. A probe light, having traversed the sample, interferes with a plane wave and constructs the interference pattern. Each interference fringe corresponds to an equi-index line of the sliced sample. The optical path-length difference between two adjacent interference fringes is equal to one wavelength, and the optical path length is defined by $n \cdot t$, where $n$ is the refractive index and $t$ is the thickness. Therefore, the index difference between two adjacent equi-index lines (interference fringes) is $\lambda / t$. Hence, since the sample is thin enough compared with the radius of the microlens, we can obtain the refractive-index distribution $n(r, z)$. From Fig. 1(b) we can see the maximum index difference $\Delta n=0.11$ and the almost hemispherical index profile of the planar microlens with nearly parabolic dependency on radius $r$ and depth $z$. Details of the index profile and ray optical property of the planar microlens will be reported in the near future.

The focused spot of the collimated $\mathrm{He}-\mathrm{Ne}$ laser beam $(0.63 \mu \mathrm{m})$ was measured with the planar microlens. We can observe an Airylike disk pattern originating from the diffraction as shown in Fig. 2(a). We show an Airy disk profile associated with a thin lens with a $0.9-\mathrm{mm}$ diam $2 a$ and a $2.9-\mathrm{mm}$ focal length $f$. The spot diameter was $7 \mu \mathrm{m}$ which is small enough in comparison with the $50-\mu \mathrm{m}$ core diam of the multimode fiber even when we use it in the long- wavelength region $(1.3-1.6 \mu \mathrm{m})$.

In Table I we show typical numerical data of a planar microlens array for the purpose of using it as a component of the stacked planar optics we shall discuss in the next section. We have confirmed that the N.A. in air (N.A. $a=n_{2} \times \sin \theta_{i}$ ) can be extended as high as 0.25 by stacking two lenses where $\theta_{i}$ is the maximum acceptance angle as shown in Table I. This value is considered large enough to use the stack as a microlens in the ordinary multimode fiber system with N.A. $=0.2$.

\section{Stacked Planar Optics}

\section{A. Basic Idea}

The stacked planar optics consists of planar optical components and the stack of them as shown in Fig. 3. All components must have the same 2-D spatial relationship which can be achieved from planar technology with the help of photolithographic fabrication of the integrated circuits. Once we align the optical axis and

adhere all the stacked components, 2-D arrayed components are realized; the mass production of axially aligned discrete components is also possible if we separate the individual components as shown in Fig. 3(c). This is the fundamental concept of the stacked planar optics which may be a new type of integrated optics.

$\lambda=0.63 \mu \mathrm{m}$
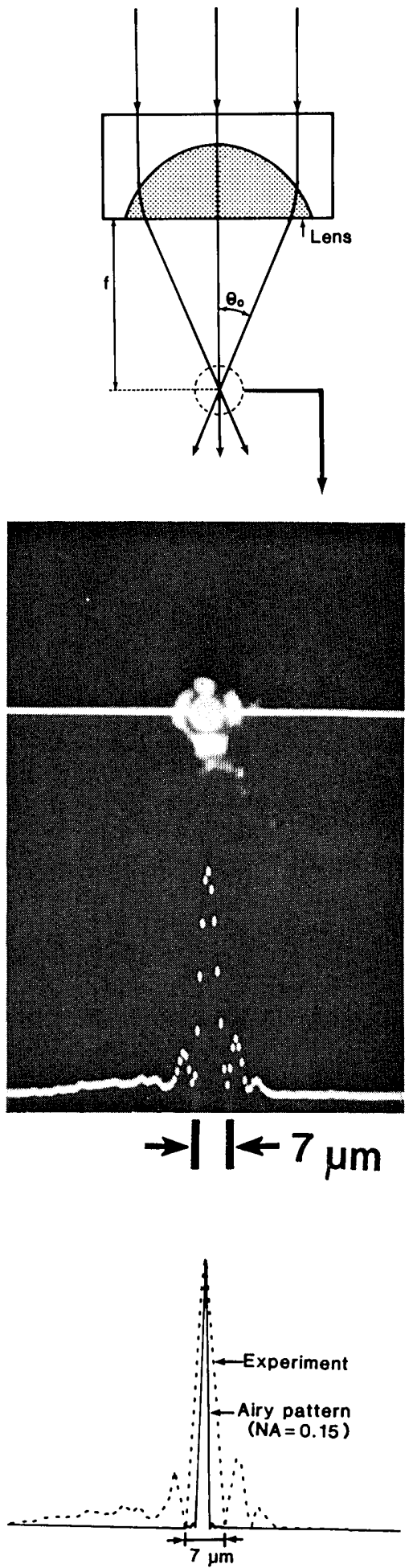

Fig. 2. Composition of stacked planar optics. 
Table I. Typlcal Numerical Data of a Planar Microlens

\begin{tabular}{|c|c|c|}
\hline & Single lens & Coupled pair \\
\hline Lens diameter & $0.9 \mathrm{~mm}$ & $0.9 \mathrm{~mm}$ \\
\hline Lens pitch & $2 \mathrm{~mm}$ & $2 \mathrm{~mm}$ \\
\hline Depth of distributed index region & $0.45 \mathrm{~mm}$ & $0.45 \mathrm{~mm}$ \\
\hline Focal length $f$ (in air) & $2.9 \mathrm{~mm}$ & \\
\hline Focal length $1_{0}$ (in substrate) & $4 \sim 4.5 \mathrm{~mm}$ & $2.5 \mathrm{~mm}$ \\
\hline Numerical Aperture (in air) & 0.15 & 0.25 \\
\hline$N A_{a}=\sin \theta_{0}=n_{2} \times \sin \theta_{i}$ & & \\
\hline Numerical Aperture (in substrate) & 0.10 & 0.17 \\
\hline$N A_{s}=\sin \theta_{i}$ & & \\
\hline Index of substrate $n_{2}$ & 1.52 & 1.52 \\
\hline Normalized index difference & 8 & 5 \\
\hline & & 10 \\
\hline
\end{tabular}

\section{B. Process}

We therefore propose a possible fabrication process for the stacked planar optics as follows: (1) design of planar optical devices (determination of thickness, design of mask shape, etc.); (2) fabrication of planar optical devices; (3) optical alignment; (4) adhesion; (5) connection of optical fibers in the case of arrayed components; and (6) separation of individual components in the case of discrete components and connection of optical fibers.

Features of the stacked planar optics include:

(1) Mass production of standardized optical components or circuits is possible, since the planar devices are fabricated by planar technology.

(2) Optical alignment is easy.

(3) Optical components of different materials (glass, semiconductors, electrooptical crystals, etc.) can be connected in tandem. This had been thought difficult in integrated optics consisting of planar optical waveguides, ${ }^{5}$ which are formed on planar substrates where the connection of different components needs highprecision optical adjustment, since the light is transmitted through a thin waveguide only a few microns in thickness and width.

(4) Coupling of optical fibers is easy, i.e., it could possibly be done without optical adjustment if precise fabrication of a 2-D array of holes is available. This will be detailed later.

\section{Planar Optical Devices}

In this section we discuss planar optical devices used in stacked planar optics. The actual component configurations are analogous to discrete distributed-index lens systems, but we pick up these as 2-D arrayed devices which exactly match the lens arrays.

\section{Circular Holes Array}

The circular holes array in a planar substrate is used to connect a fiber with a stacked planar optical circuit as shown in Fig. 4. The diameter of a hole is selected equal to the o.d. of the fiber. The position of each hole must be matched to that of the arrayed planar microlens to align the optical axis. The alignment process, therefore, can be achieved automatically.

\section{Aperture and Spatial Frequency Filter}

Figure 5(a) shows windows opened on a thin film evaporated on a planar substrate. These windows are used as aperture stops.

Figure 5(b) shows spatial frequency filters set on the Fourier plane that limit the spatial frequency. These devices work to eliminate useless light and to filter-out unwanted modes. The lithographic technique can be
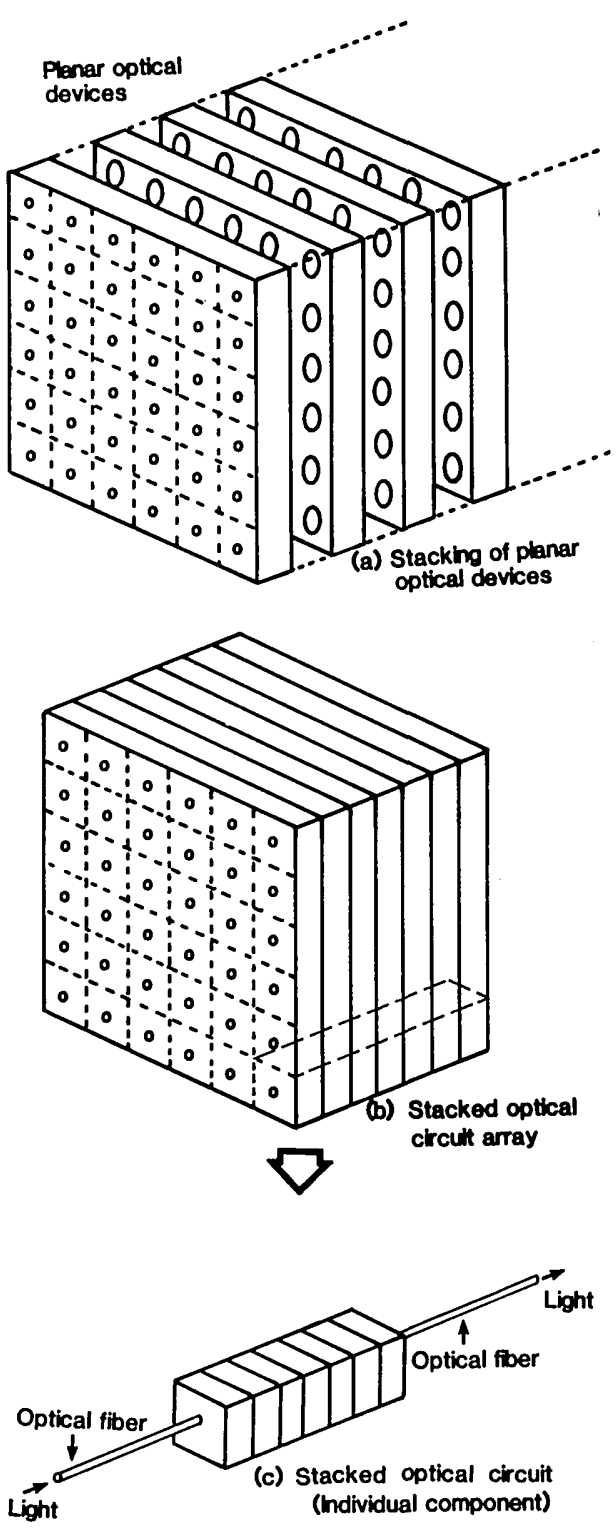

Fig. 3. Focused spot by a planar microlens; the He-Ne laser beam was illuminated from the substrate side and the focused spot in air was observed. 


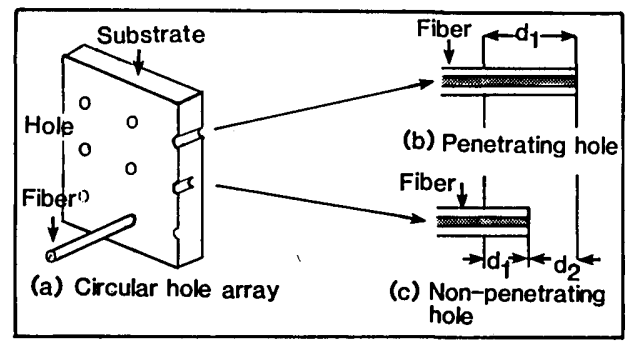

Fig. 4. Array of circular holes.

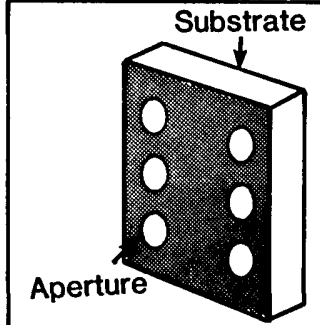

(a) Aperture array

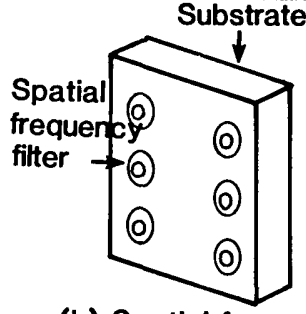

(b) Spatial frequency filter array

Fig. 5. Aperture and spatial frequency filter.

used to fabricate these components on a substrate or back surface of the planar microlens.

\section{Wavelength Filter and Mirror}

A mirror and a wavelength filter on a planar substrate are well known and widely used in conventional optics and optical circuits in optical fiber communication systems. ${ }^{1-3}$ In stacked planar optics, these devices are prepared in a single-batch process with many of the arrayed components.

\section{Polarizer and Phase Plate}

In a stacked planar optical circuit, a polarizer, analyzer, $\lambda / 2$ phase plate, and $\lambda / 4$ phase plate can be stacked easily.

\section{Active Optical Components}

The component with electrooptic effect and magnetooptic effect can be applied to deflectors, switches, modulators, and unidirectional waveguides. Although the arrangement of the necessary electrodes sometimes may prevent construction of a 2-D array, at least a 1-D array with a stacked configuration can be profitably mass-produced. Since the structure of each active device has a special configuration, custom design is called for.

\section{Large N.A. Lens Array}

Since the radiating angle of light from a semiconductor laser diode (LD) and a light emitting diode (LED) is large $\left(40^{\circ}\right.$ for $\mathrm{LD}$ and $90^{\circ}$ for LED), we need a large numerical aperture lens to accept the light effectively. An arrayed lens with a curved surface, formed from precision cast plastic or glass and adhered semispheres on a substrate, may be used although not of planar structure.

Table II. Basic Components of Stacked Planar Optics and Applied Components

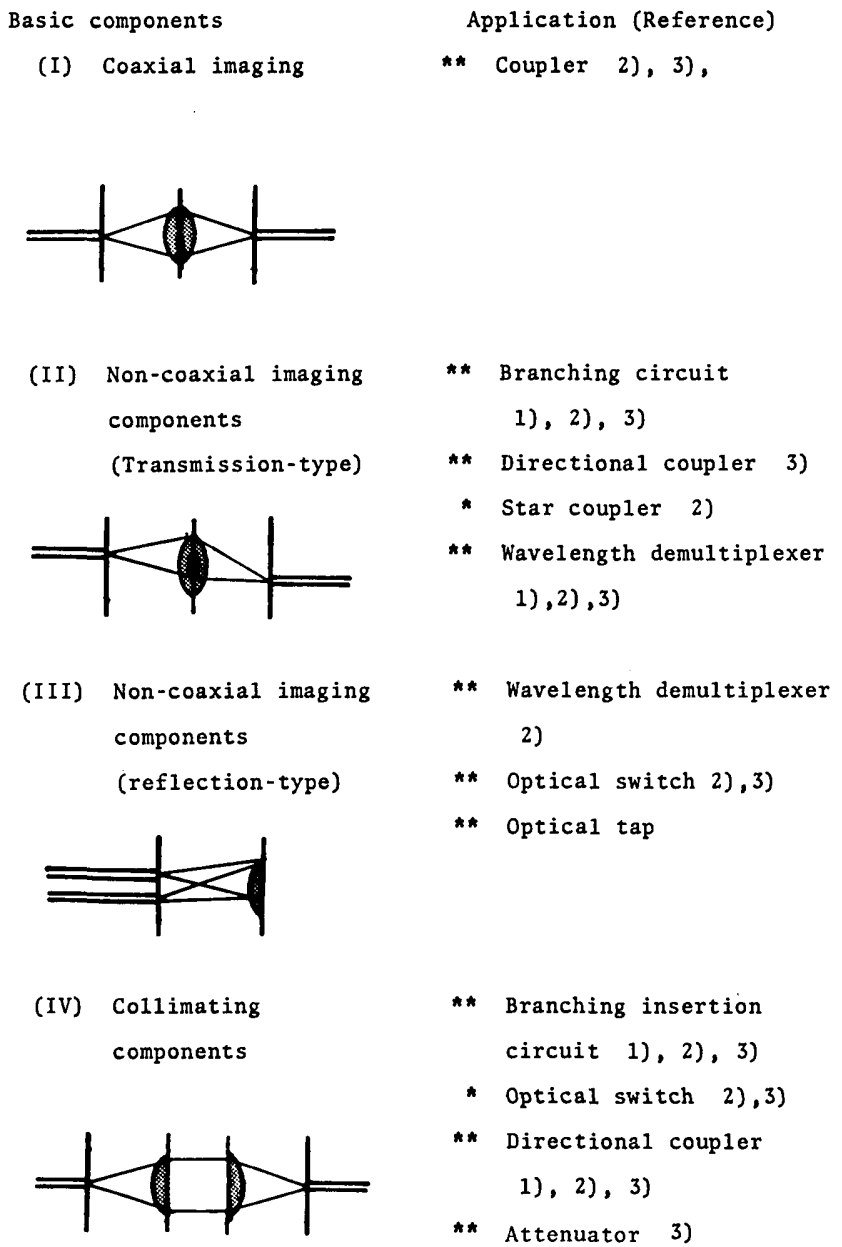

(II) Non-coaxial imaging components (Transmission-type)

(III) Non-coaxial imaging components (reflection-type)

(IV) Collimating components

** Branching insertion circuit 1), 2), 3)

* Optical switch 2),3)

** Directional coupler 1), 2), 3)

\#* Attenuator 3) 


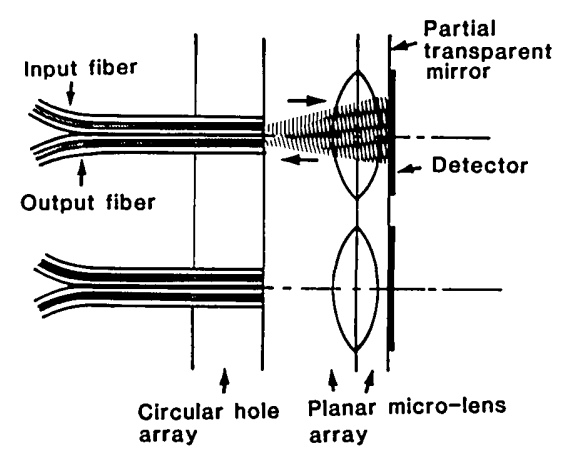

Fig. 6. Optical tap array with stacked planar optics configuration.

\section{Applications}

As summarized in Table II, many kinds of optical circuit can be integrated in the form of stacked planar optics, where ** denotes a circuit that can be integrated in a 2-D array and * denotes a circuit that can be integrated in a 1-D array. We do not detail optical circuits; readers are referred to Ref. 2.

We have tested the possibility of realizing stacked planar optics by making the four-channel optical tap array shown in Fig. 6 . This component has a function such that the light from one fiber is collimated by the planar microlens and reflected by the mirror on the back surface and refocused onto the other output fiber. ${ }^{4}$ The planar microlenses array was stacked to increase the N.A. The thickness of the tap array is $\sim 3 \mathrm{~mm}$ to couple the light from the input fiber to the output fiber on the front surface. On the back surface the partially transparent mirror was evaporated. The transmittance of the mirror to the detector was $4.7 \%$. Some of the light is monitored with the detector which is prepared on the back surface. The coupling efficiency of the reflected light was $52 \%$. The position of the fibers and the thickness of the substrate is not yet optimized. The coupling efficiency could be improved by solving these problems and by reducing the aberrations of the planar microlens.

In this paper we do not deal with coupling effects among optical components in a stacked planar optical circuit. However, it is possible to construct a 3-D optical circuit which forms a network structure by allowing coupling among adjacent components.

Since the accumulation of lens aberrations may bring about coupling loss, the number of stacks is limited by aberration of the planar microlenses. Reduction of the aberrations in the planar microlens is important, therefore, when using a stacked planar optics with a large number of stacks. This problem will be discussed elsewhere.

\section{Summary}

We have proposed a new concept for the integration of optical circuits, i.e., stacked planar optics. Using the stacked planar optics not only is the monolithic fabrication of optical circuits, such as directional coupler, wavelength demultiplexer, possible but also is construction of 3-D optical circuits by allowing coupling between individual components of an appropriately designed array.

\section{References}

1. N. Kobayashi, T. Ishikawa, K. Minemura, and S. Sugimoto, Fiber Integrated Opt. 2, 1 (1979).

2. S. Sugimoto, K. Kobayashi, and S. Matsushita, J. IECE Jpn. 61, 1114 (1978); S. Sugimoto, R. Ishikawa, and K. Kobayashi, Optics 10, 128 (1981) (Japanese).

3. W. J. Tomlinson, Appl. Opt. 19, 1127 (1980).

4. J. J. Pan, in Digest of Conference on Lasers and Electrooptics Systems (Optical Society of America, Washington, D.C., 1976), paper THE3.

5. S. E. Miller, Bell Syst. Tech. J. 48, 2059 (1969).

6. K. Iga, M. Oikawa, Y. Misawa, and J. Banno, in Digest of Topical Meeting on Integrated and Guided-Wave Optics (Optical Society of America, Washington, D.C., 1982), paper FB6.

7. M. Oikawa, K. Iga, and T. Sanada, Jpn. J. Appl. Phys. 20, L51 (1981).

8. M. Oikawa, K. Iga, T. Sanada, N. Yamamoto, and K. Nishizawa, Jpn. J. Appl. Phys. 20, L296 (1981).

9. M. Oikawa, K. Iga, and T. Sanada, Electron. Lett. 17, 452 (1981).

10. G. D. Khoe, H. G. Kock, J. A. Luijendik, C. H. J. van en Brekel, and D. Kueppers, in Technical Digest, Seventh European Conference on Optical Communications, Copenhagen (1981), paper 7.6.

11. Y. Kokubun and K. Iga, in Digest of Topical Meeting on Gradient Index Optics (Optical Society of America, Washington, D.C., 1981), paper MD4; Appl. Opt. 21, 1030 (1982).

We thank Y. Suematsu and T. Tako of the Tokyo Institute of Technology for support, and I. Kitano, T. Yamazaki, K. Nishizawa, and N. Yamamoto of the Nippon Sheet Glass Co. for discussions. 